\title{
Disruption of intraflagellar protein transport in photoreceptor cilia causes Leber congenital amaurosis in humans and mice
}

\author{
Karsten Boldt,, ${ }^{1,2}$ Dorus A. Mans,,3,4 Jungyeon Won, ${ }^{5}$ Jeroen van Reeuwijk,3,4 Andreas Vogt,,1,2 \\ Norbert Kinkl, ${ }^{1,2}$ Stef J.F. Letteboer, ${ }^{3,4}$ Wanda L. Hicks, ${ }^{5}$ Ron E. Hurd, 5 Jürgen K. Naggert, ${ }^{5}$ \\ Yves Texier, ${ }^{1,2}$ Anneke I. den Hollander, ${ }^{3,4,6,7}$ Robert K. Koenekoop, ${ }^{8}$ Jean Bennett, ${ }^{9,10}$ \\ Frans P.M. Cremers, ${ }^{3,4}$ Christian J. Gloeckner, 1,2 Patsy M. Nishina, 5 \\ Ronald Roepman, ${ }^{3,4,7}$ and Marius Ueffing ${ }^{1,2}$
}

\begin{abstract}
${ }^{1}$ Division of Experimental Ophthalmology and Medical Proteome Center, Center of Ophthalmology, University of Tübingen, Tübingen, Germany. 2Department of Protein Science, Helmholtz Zentrum München, German Research Center for Environmental Health, Neuherberg, Germany. ${ }^{3}$ Department of Human Genetics, Radboud University Nijmegen Medical Centre, Nijmegen, Netherlands. ${ }^{4}$ Nijmegen Centre for Molecular Life Sciences, Radboud University Nijmegen, Nijmegen, Netherlands. ${ }^{5}$ The Jackson Laboratory, Bar Harbor, Maine, USA. ${ }^{6}$ Department of Ophthalmology, Radboud University Nijmegen Medical Centre, Nijmegen, Netherlands. ${ }^{7}$ Institute for Genetic and Metabolic Disease, Radboud University Nijmegen, Nijmegen, Netherlands. ${ }^{8}$ McGill Ocular Genetics Center, McGill University Health Center, Montreal, Quebec, Canada. ${ }^{9}$ Scheie Eye Institute,

F.M. Kirby Center for Molecular Ophthalmology, University of Pennsylvania School of Medicine, Philadelphia, Pennsylvania, USA. ${ }^{10}$ Center for Cellular and Molecular Therapeutics, Children's Hospital of Philadelphia, Philadelphia, Pennsylvania, USA.
\end{abstract}

\begin{abstract}
The mutations that cause Leber congenital amaurosis (LCA) lead to photoreceptor cell death at an early age, causing childhood blindness. To unravel the molecular basis of LCA, we analyzed how mutations in LCA5 affect the connectivity of the encoded protein lebercilin at the interactome level. In photoreceptors, lebercilin is uniquely localized at the cilium that bridges the inner and outer segments. Using a generally applicable affinity proteomics approach, we showed that lebercilin specifically interacted with the intraflagellar transport (IFT) machinery in HEK293T cells. This interaction disappeared when 2 human LCA-associated lebercilin mutations were introduced, implicating a specific disruption of IFT-dependent protein transport, an evolutionarily conserved basic mechanism found in all cilia. $L c a 5$ inactivation in mice led to partial displacement of opsins and light-induced translocation of arrestin from photoreceptor outer segments. This was consistent with a defect in IFT at the connecting cilium, leading to failure of proper outer segment formation and subsequent photoreceptor degeneration. These data suggest that lebercilin functions as an integral element of selective protein transport through photoreceptor cilia and provide a molecular demonstration that disrupted IFT can lead to LCA.
\end{abstract}

\section{Introduction}

Leber congenital amaurosis (LCA; OMIM 204000) is the most severe hereditary retinal dystrophy. It is characterized by early visual loss, sensory nystagmus, amaurotic pupils, and absence of scotopic and photopic electroretinogram (ERG) responses before 1 year of age. Mutations in at least 15 genes lead to LCA (1-3). Despite this genetic heterogeneity, the clinical features of LCA are remarkably consistent. This clinical fact points to overlapping pathogenic disease mechanisms arising from different cellular insults. A recently identified group of LCA-associated proteins - LCA5-encoded lebercilin (4), CEP290, RPGRIP1, and TULP1 - localizes to connecting cilia of photoreceptor cells. The connecting cilium (CC) is a specialized ciliary transition zone that supports selective transport of proteins and membrane vesicles, also known as intraflagellar transport (IFT), between the protein-producing inner segment (IS) and the photoreceptive outer segment (OS) of the photoreceptor cell (3). It involves 2 directional, highly conserved transport mechanisms along the ciliary microtubule tracks, essentially required in cilia of all species for the assembly

Authorship note: Karsten Boldt, Dorus A. Mans, and Jungyeon Won contributed equally to this work, as did Ronald Roepman and Marius Ueffing.

Conflict of interest: The authors have declared that no conflict of interest exists. Citation for this article: J Clin Invest. 2011;121(6):2169-2180. doi:10.1172/JCI45627. of cilia (anterograde transport, toward the ciliary tip) as well as for recycling of axonemal turnover products (retrograde transport, in the opposite direction; refs. 5, 6). Studies in Chlamydomonas have suggested that anterograde transport is mediated by particles consisting of a multisubunit protein complex (IFT complex B proteins), driven by the kinesin-II motor proteins. Similarly, retrograde transport is mediated by the IFT complex A particle, driven by the cytoplasmic dynein $2 / 1 \mathrm{~b}$ motor proteins $(7-10)$. Because of the immense turnover rate of the OSs of photoreceptors as a result of the highly active phototransduction cascade, about $10 \%$ of this compartment is shed daily at the photoreceptor apex and phagocytosed by the RPE cells (11). This unique and rapid recycling of what is basically the photoreceptor sensory cilium requires a particularly active IFT in photoreceptor cells.

Retinas of mutant mouse models for LCA-associated RPGRIP1 (Rpgrip $1^{\text {nmf247}}$; ref. 12) and CEP290 (rd16; ref. 13) display mislocalization of phototransducing proteins in combination with photoreceptor function loss and rapid photoreceptor degeneration. Such mislocalization of proteins makes it tempting to hypothesize that this is the result of defective IFT. This, then, may underlie the observed pathogenesis of photoreceptor degeneration in these and other LCA mutant mouse models, as well as in LCA patients. To date, however, molecular evidence to confirm the defective IFT hypothesis in LCA remains elusive. 

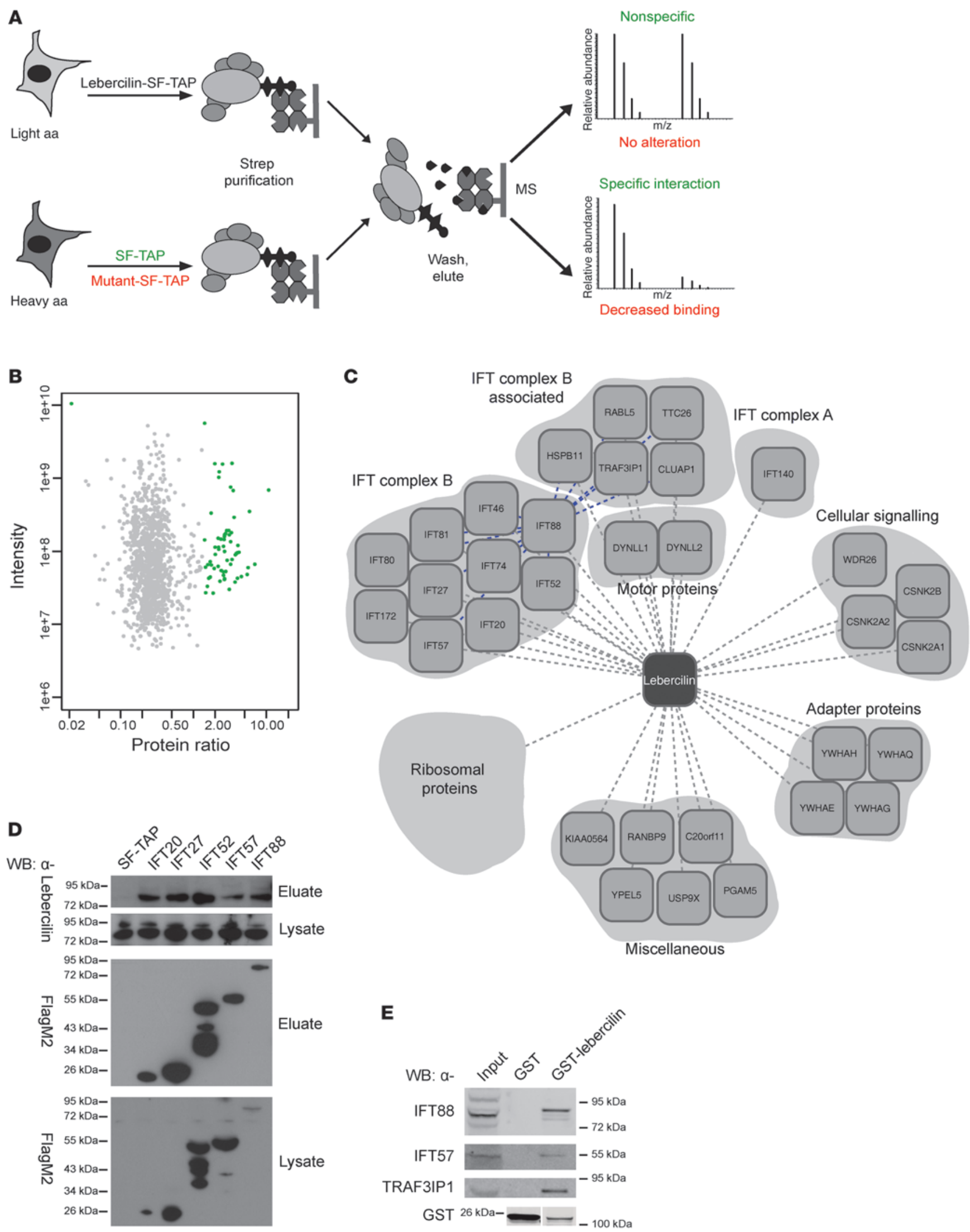


\section{Figure 1}

Quantitative protein complex analysis of lebercilin. (A) SILAC/AP approach to detect specific protein complex components. Identification of significantly enriched proteins compared with control (SF-TAP; green), and analysis of the effect of 2 LCA-causative mutations in lebercilin (p.P493TfsX1 and p.Q279X) on protein complex formation, by comparing the lebercilin complex with complexes formed by the mutants (red) in SILAC-labeled HEK293T cells. MS, mass spectrometry. (B) Detection of specific protein complex components. Plotted are $\log _{10}$ ratios and $\log _{10}$ intensities for each protein identified and quantified in at least 2 of 3 biological replicates. Significantly enriched proteins $(P<0.001)$ are plotted in green. (C) Protein complex of wildtype lebercilin. Gene names for each protein are shown (see Supplemental Table 1). Blue lines denote interconnectivity, as determined by IFT88 SF-TAP analysis (Supplemental Table 3). (D) Confirmation of IFT-lebercilin association by detecting endogenous lebercilin in eluates of IFT20, IFT27, IFT52, IFT57, and IFT88 SF-TAP experiments. Shown are the lysates as control for lebercilin input. All SF-TAP tagged constructs were expressed and purified, as demonstrated by Western blot using anti-FlagM2. Lebercilin was detected by anti-lebercilin (SN2135) in all eluates, but not in the SF-TAP control, confirming the SILAC/AP results. (E) Confirmation of the association of IFT and IFTassociated proteins with lebercilin in retina by detecting endogenous IFT88, IFT57, and TRAF3IP1 in a GST pulldown of lebercilin, but not in the GST control. Both GST-lebercilin and GST alone were expressed. Lanes for the anti-GST blot were run on the same gel but were noncontiguous (white line).

In the present study, we used a powerful combination of a quantitative affinity proteomics approach using LCA5-encoded lebercilin and 2 human LCA-causative mutations in lebercilin, together with the development and detailed examination of a homozygous Lca5 gene trap mouse model ( $\left.L c a 5^{g t / g t}\right)$. This procedure, which can be similarly used to functionally assess other genetic disorders, has instantly yielded a detailed view of the LCA5 disease mechanism. We determined that lebercilin physically interacted with a nearly complete IFT complex B particle, as well as with some IFT complex A proteins. As $L C A 5$-associated mutations abrogated this interaction, our results demonstrated a direct involvement of defective IFT in the LCA disease phenotype. The phenotype we observed in the Lca5gt/gt mice, an early-onset defect in the development of complete OSs and failure to fully and correctly (trans)locate arrestin and opsin, was fully in line with the proposed disease mechanism.

\section{Results}

Lebercilin physically interacts with IFT proteins. In order to provide a mechanistic view of the molecular perturbations in LCA, we developed a proteomics-based workflow to analyze the lebercilin interactome on a quantitative level with greatly increased sensitivity. We combined affinity purification (AP) with stable isotope labeling of aa in cell culture (SILAC; 14, 15), followed by quantitative mass spectrometry and bioinformatic analysis (16). Comparison of the resulting profiles for wild-type and mutated lebercilin (Figure 1A) allowed us to quantitatively and comparatively assess changes within a protein complex, caused by allelic variants, in this case of mutations in LCA5 (4).

To identify the components of the lebercilin protein complex with high sensitivity, we expressed lebercilin fused to the Strep-tag II/FLAG tandem AP tag (SF-TAP) as well as the SF-TAP alone as a negative control in either heavy- or light-isotope SILAC-labeled HEK293T cells. Both cell populations were subjected to a quick 1-step AP to increase the sensitivity for labile and weakly associ- ated components. The samples were combined after the purification step. The combined samples, discriminated by incorporated heavy or light isotopes, were then subjected to quantitative mass spectrometric analysis. After software-based quantification, proteins significantly enriched in the lebercilin sample $(P<0.001)$ were considered to be specific components of the lebercilin protein complex and were grouped according to their proposed function and visualized by Cytoscape-assisted representation (Figure 1, B and $C$, and Supplemental Table 1; supplemental material available online with this article; doi:10.1172/JCI45627DS1). By using this SILAC/AP approach in HEK293T cells, we not only confirmed the association of proteins we had previously identified by SF-TAP (4), but identified almost all Chlamydomonas IFT orthologs (17) in the lebercilin protein complex (Figure 1C and Supplemental Table 1). We validated the association of IFT proteins with lebercilin by detecting endogenous lebercilin in SF-TAP eluates of 5 IFT proteins tested by Western blot (Figure 1D). Additionally, by GST pulldown of exogenous lebercilin in bovine retina, we were able to detect endogenous IFT proteins (Figure 1E), which indicates that the physical association of lebercilin with IFT proteins is present in retina as well. Furthermore, we identified IFT complex B-associated proteins (CLUAP1, HSPB11, RABL5, TRAF3IP1, and TTC26; Figure 1, C and E) as lebercilin interaction partners, the orthologs of which have previously been shown to be involved in IFT in Trypanosoma (18), nematode (19-23), and fish (24) and to form protein complexes with IFT protein complex B of Chlamydomonas (25) or mouse (26). A list of known human IFT and IFT-associated proteins is included in Supplemental Table 2. SF-TAP analysis of IFT complex B subunit IFT88 further showed that virtually all IFT complex B-associated proteins were part of the IFT88 complex. This demonstrated, for the first time to our knowledge, the existence of the complete IFT complex B particle as a physical IFT module in mammalian cells (Supplemental Table 3).

LCA-associated mutations in lebercilin disrupt the physical connection with IFT. The effect of LCA mutations on the lebercilin interactome was assessed by quantitative protein complex comparisons of wild-type lebercilin with 2 known human LCA5 mutations (Figure 1A). The same setup was used as for the detection of specific protein complex components, with a single modification. Instead of SF-TAP as control, lebercilin-p.P493TfsX1 and lebercilin-p.Q279X were expressed, and their complexes were compared with normal lebercilin in HEK293T cells. The correct production of both mutants was demonstrated by Western blot analysis of HEK293T lysates (Supplemental Figure 1A) as well as by the identification of peptides covering large parts of the theoretical sequence (Supplemental Figure 6, A and B). Both mutations are causative for LCA without obvious phenotypic differences (4). p.P493TfsX1 is located in the last exon of LCA5, and therefore the mRNA should not be subjected to nonsense-mediated mRNA decay (27). This was confirmed by real-time quantitative PCR (qPCR) analysis of EBV-transformed lymphocytes from a LCA patient harboring a homozygous p.P493TfsX1 mutation in LCA5 (family 27240; ref. 4), which showed no significant decrease in LCA5 mRNA expression compared with EBV-transformed lymphocytes of a control subject (Supplemental Figure 1B). Our analyses showed that both mutations resulted in the loss of all identified IFT and IFT-associated proteins from the protein complex (Figure 2, Supplemental Table 1, and Supplemental Figure 1C). These results were confirmed by Western blot analysis, in which IFT74 and IFT88 could not be detected in SF-TAP 
A

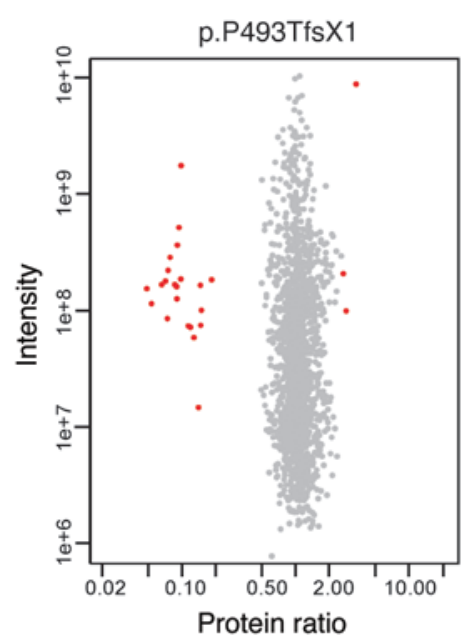

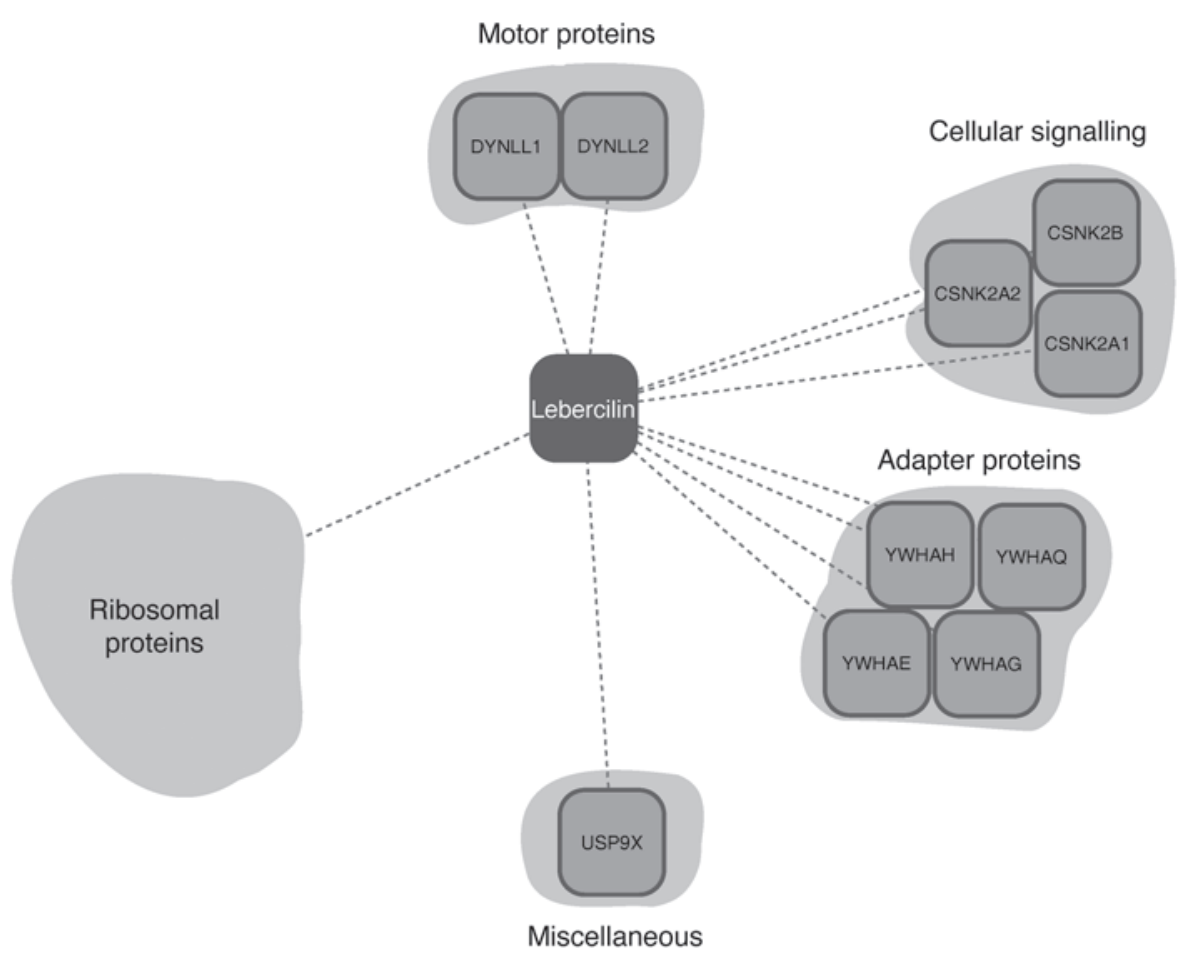

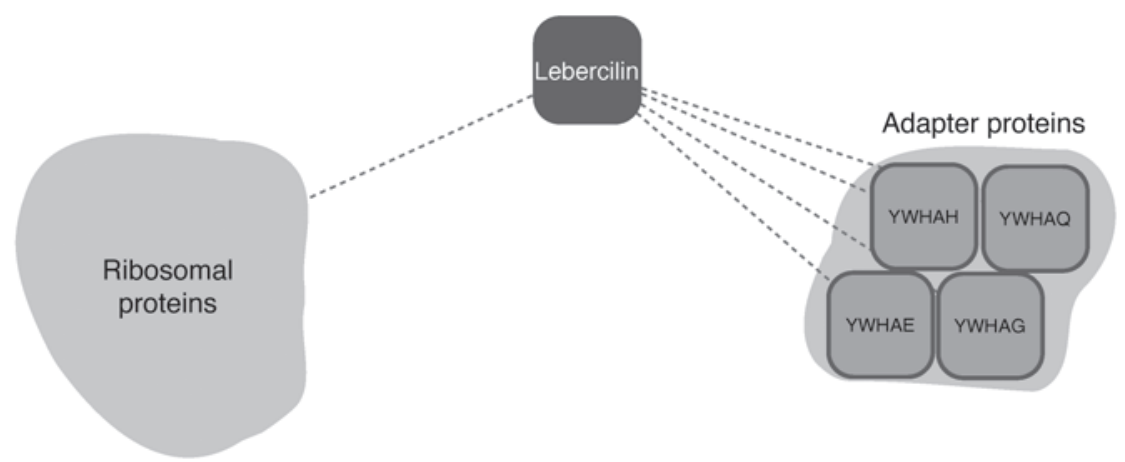

Motor proteins

iscellaneous
B

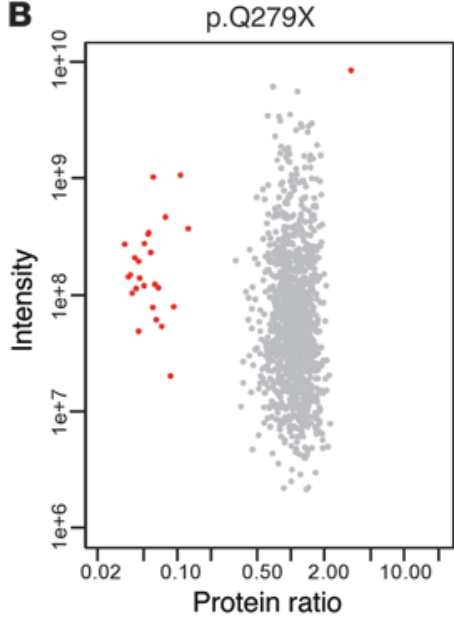

\section{Figure 2}

Quantitative protein complex analysis of LCA-causative lebercilin mutations. Detection of protein complex alterations by comparison of the complexes of SF-TAP tagged lebercilin to either lebercilin-p.P493TfsX1 (A) or lebercilin-p.Q279X (B) in HEK293T cells. Plotted are log 10 ratios and $\log _{10}$ intensities as quantified by MaxQuant from 3 independent experiments. For each experiment, label switching was done to exclude label-specific effects. Shown are only proteins quantified and identified in at least 2 of 3 experiments. Highly significant $(P<0.001)$ altered protein interactions are plotted in red, the majority of them being IFT proteins (Supplemental Table 1). Also shown are representations of the respective protein complexes; all proteins of the IFT protein complex, as well as WDR26, KIAA0564, PGAM5, YPEL5, C20orf11, and RANBP9, were lost for both mutants. CSNK2A1/2, CSNK2B, DYNLL1, DYNLL2, and USP9X only lost association to lebercilin-p.Q279X, not to lebercilin-p.P493TfsX1.

eluates of p.P493TfsX1 and p.Q279X, whereas the presence of 14-3-3e was not altered (Supplemental Figure 1A). Moreover, 6 other proteins previously identified in the lebercilin complex (4) were lost from the complex of both lebercilin mutants: WDR26, KIAA0564, PGAM5, YPEL5, C20orf11, and RANBP9 (Supplemental Table 1). Therefore, these may also be associated to lebercilin function. The more aminoterminally truncated mutant p.Q279X additionally displayed reduced binding to CSNK2A1/2, CSNK2B, DYNLL1, DYNLL2, and USP9X (Figure 2B), which suggests that this protein variant has lost additional protein binding domains. This stresses the role of the carboxyterminal part of lebercilin as a crucial and specific interaction domain for IFT proteins. 
A

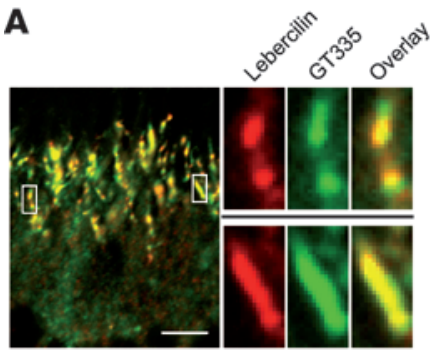

C

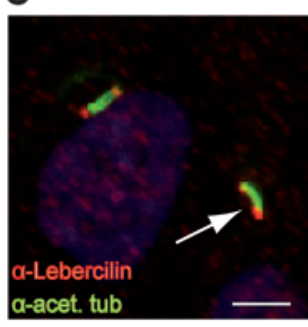

E

Non-targeting siRNA
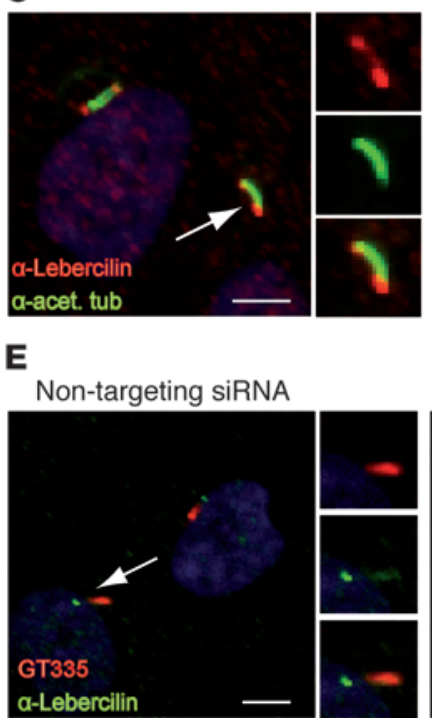

B

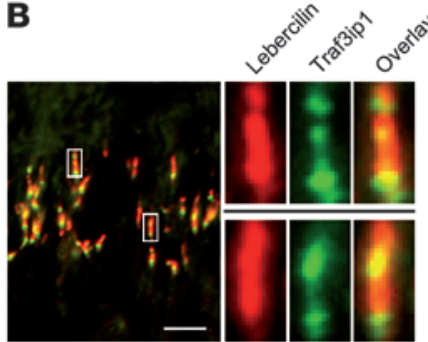

D

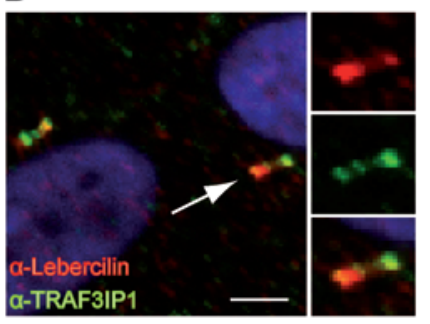

LCA5 SIRNA

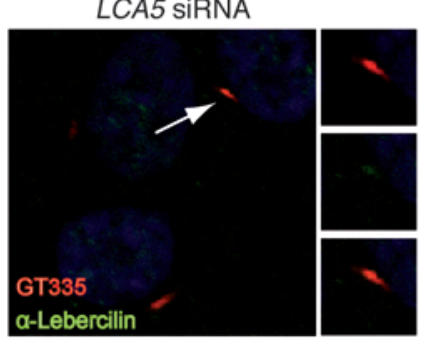

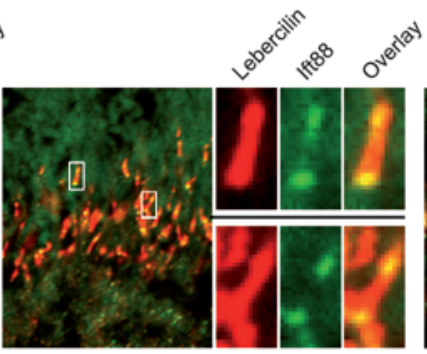
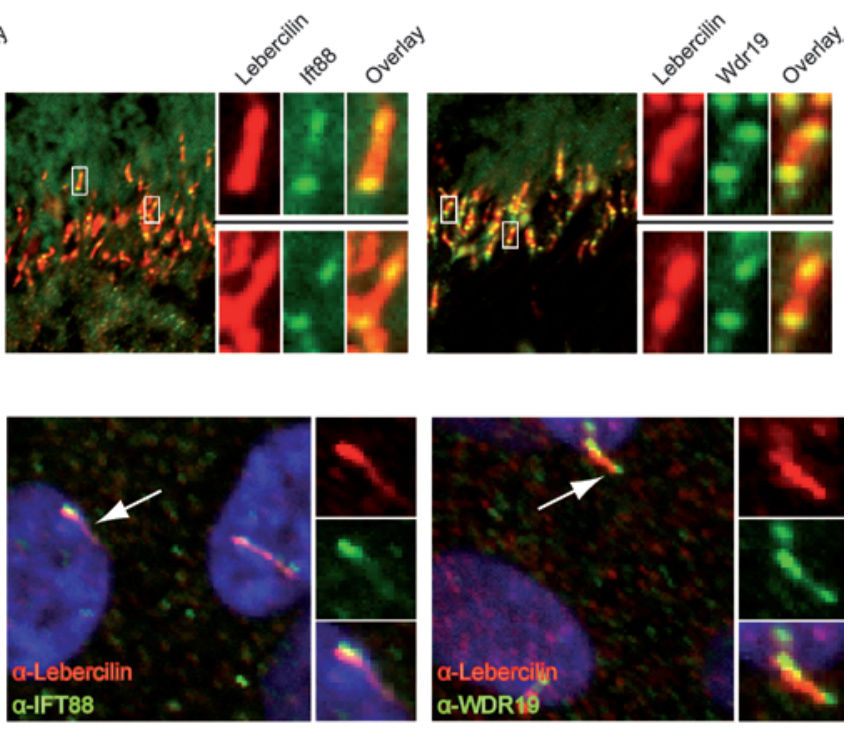

$\mathbf{F}$
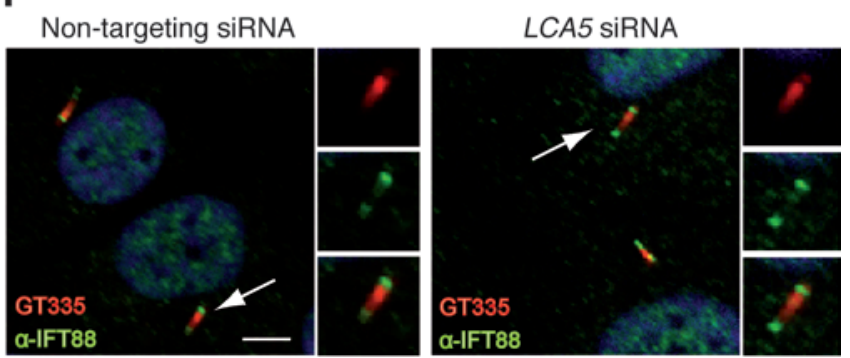

Figure 3

Endogenous localization of lebercilin and IFTs in mammalian retina and retinal cells. (A) Immunohistochemistry of mouse BALB/c retina (P20) showed endogenous lebercilin (SN2134) expression in the connecting cilia of mouse photoreceptors, where it colocalized with the ciliary marker polyglutamylated tubulin (GT335). (B) Endogenous lebercilin (SN2134) colocalized with endogenous IFT complex B proteins Traf3ip1, Ift88, and IFT complex A protein Wdr19 in connecting cilia of mouse photoreceptors. (C) Immunocytochemistry of hTERT-RPE1 cells confirmed lebercilin (SN2134) localization in primary cilia (arrow). (D) Colocalization of endogenous lebercilin (SN2134) in the cilium with endogenous TRAF3IP1, IFT88, and WDR19 (arrows). (E) siRNA-mediated knockdown of LCA5 did not affect ciliogenesis compared with nontargeting siRNA-transfected hTERTRPE1 cells (arrows). GT335 stained the primary cilium. SN2134 antibody was used to stain lebercilin. (F) Knockdown of LCA5 in hTERT-RPE1 cells did not affect ciliary localization of IFT88 (arrows). Scale bars: $5 \mu \mathrm{m}(\mathbf{A}-\mathbf{F})$. Enlarged views are shown in the insets $(\mathbf{A}$ and $\mathbf{B}, \times 5 ; \mathbf{C}-\mathbf{F}, \times 2)$.

Lebercilin and IFT proteins colocalize in cilia of retinal cells. To validate the interaction between endogenous lebercilin and IFT proteins in retinal cells, we analyzed the localization of lebercilin and IFT proteins in mouse retina and in ciliated human hTERT-RPE1 cells. Lebercilin localized along the CC of mouse photoreceptors (Figure $3 \mathrm{~A}$ ) and in primary cilia of hTERT-RPE1 cells (Figure 3C). Colocalization between lebercilin and IFT complex $A$ and $B$ proteins was observed in the CC of mouse photoreceptors (Figure 3B) and in primary cilia of hTERT-RPE1 cells (Figure 3D).

Lebercilin is not a structural component of the IFT machinery. We performed knockdown of TRAF3IP1, which plays a critical role in cilium assembly in Caenorbabditis elegans (28), to determine whether IFT is involved in the ciliary localization of lebercilin. siRNA-mediated knockdown of TRAF3IP1 in hTERT-RPE1 cells affected ciliogenesis, as well as subsequent ciliary lebercilin localization, compared with nontargeting siRNA-transfected cells (Supplemental Figure 2C). Knockdown of LCA5 in hTERT-RPE1 cells, however, did not affect ciliogenesis or ciliary localization of IFT88 (Figure 3, E and F), which indicates that lebercilin and lebercilin-associated proteins are likely to be essential for specialized transport by the IFT machinery, rather than a structural component of the IFT core.

Inactivation of Lca5 in mice recapitulates LCA in humans. To investigate the role of lebercilin in IFT-mediated transport in the retina, we generated mice bearing an inactivating gene trap insertion in intron 3 of Lca5 (Supplemental Figure 3, A and B). Lca5 inactivation in the homozygous $L c a 5^{g t / g t}$ mice was confirmed at the RNA and protein levels (Supplemental Figure 3, C-F). $\beta$-Galactosidase staining allowed us to detect $L c a 5$ expression in ciliated tissues of heterozygous $L c a 5^{g t /+}$ mice (Supplemental Figure 4, A-F). Homozygous F2 Lca5t/gt mice were viable up to 1 year of age, the latest time point examined. Mutant males and females both sired offspring successfully. Phenotypically, Lca $5^{g t / g t}$ mice developed retinal patches of depigmentation and lacked rod and cone ERG responses to light stimuli at a very early stage (Figure 4, A and B), thereby recapitulating LCA in humans. Despite the broad expression of Lca5 in ciliated tissues (13), the disease phenotype was restricted to the retina; brain histology, hearing assessed by auditory brainstem 


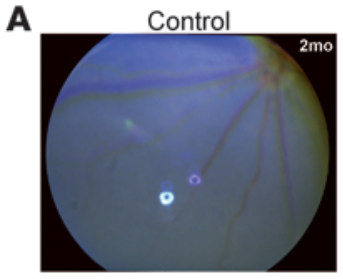

Lca5 ${ }^{\text {gtgt }}$

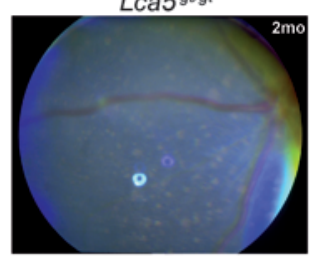

C

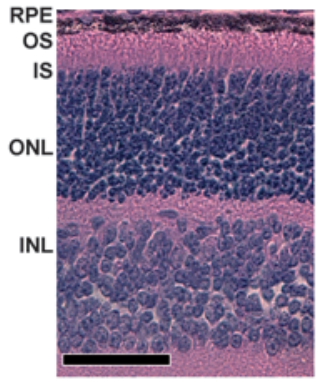

P12

- Control

D

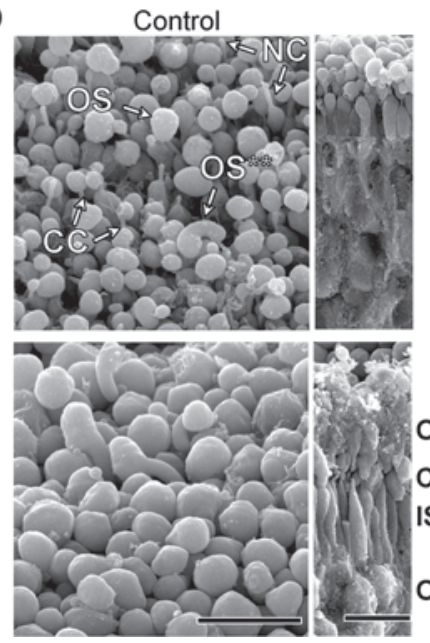

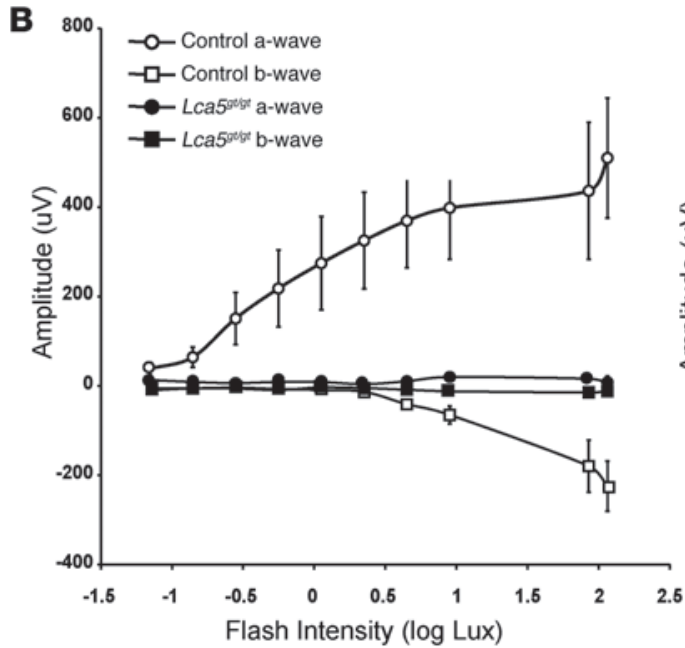

Flash Intensity (log Lux)
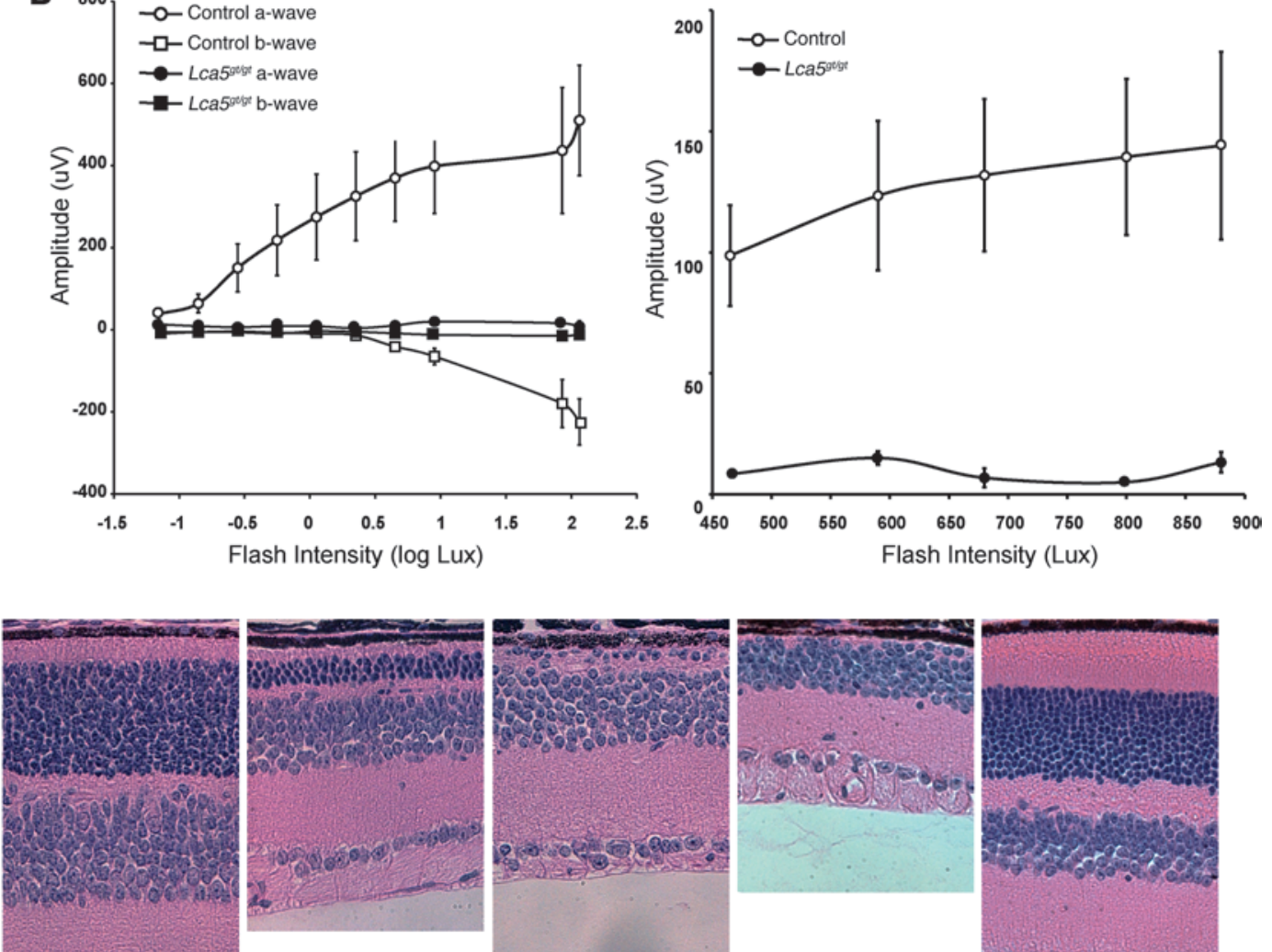

P12

P28
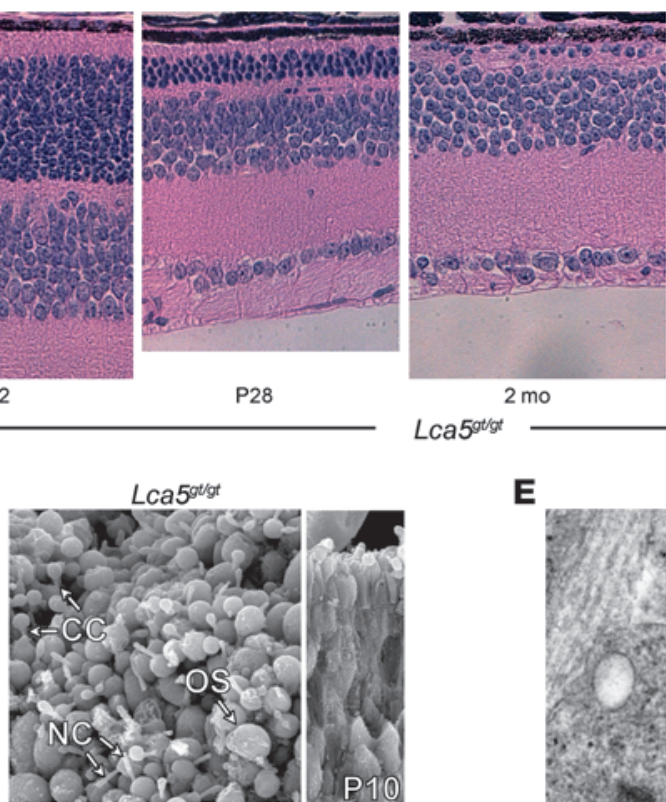

$2 \mathrm{mo}$

Lca5 $5^{\text {tht }}$

E

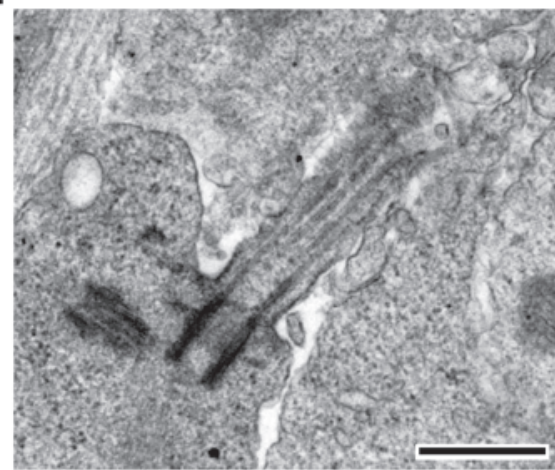

Figure 4

Clinical and morphological assessment of Lca5gt/gt mouse retina. (A) Fundus photography of Lca5 ${ }^{g t / g t}$ animals and littermate controls. At 2 months of age, depigmented patches were observed. (B) Scotopic and photopic ERG amplitudes of rod (left) and cone (right) waveforms at P25 $(n=3)$. Data are mean \pm SEM. (C) Progressive retinal degeneration demonstrated in Lca5 ${ }^{g t / g t}$ mice. Retinal sections of $L c a 5^{g t / g t}$ mice and wild-type controls, between P12 and 4 months of age, stained with hematoxylin and eosin. At P12, the OS/IS layers were remarkably thinner in Lca5gt/gt animals, whereas the ONL and INL were comparable to those of controls. At P28, the photoreceptor nuclei in the ONL were significantly reduced compared with controls. By 4 months of age, the photoreceptor layer was completely absent in Lca5gtgt mice. (D) Scanning EM of control and $L c a 5^{\text {gt/gt }}$ retinas at P10 and P15 from a scleral orientation (left) and fractured view (right). Control OSs at P10 were ovoid and regular in size. Vertically stacked OS discs (double asterisks) were rarely observed. OSs in Lca5gt/gt retinas were shorter and irregular in size and shape. By $\mathrm{P} 15$, OS discs were disorganized and did not reach the length observed in controls. Most OSs were rudimentary or abnormal in shape (asterisk). (E) Transmission EM of Lca5gt/gt retinas showed that the structure of the CC, including basal body, was preserved at P8. RPE, retinal pigment epithelium; NC, nascent cilia. Scale bars: $50 \mu \mathrm{m}$ (C); $10 \mu \mathrm{m}$ (D); $500 \mathrm{~nm}$ (E). 
response (ABR), and olfactory function were similar to those of controls (data not shown). The fundus of Lca5 heterozygous animals was normal at all ages examined (data not shown).

Lca5 inactivation results in shorter ISs and OSs. The retinal phenotype resulting from $L c a 5$ inactivation was studied in greater detail using histology and EM. Retinal sections of Lca $5^{g t / g t}$ mice at P12 showed shorter ISs and OSs, whereas the outer nuclear layer (ONL) was unaffected. At 4 months of age, the photoreceptor layer was completely absent in these animals (Figure 4C). Using transmission EM and scanning EM, we observed that the initiation of OS development in Lca5 ${ }^{\text {st/gt }}$ was delayed and OSs were disorganized (Figure 4D); discs in the OS were not oriented properly at P9-P13 (Supplemental Figure 5A). The structure of both the CC and the basal body appeared to be normal at this stage (Figure 4, D and E, and Supplemental Figure 5A).

Phototransduction proteins mislocalize in photoreceptors upon Lca5 inactivation. The transport of phototransduction proteins along the CC between the IS and OS is crucial for photoreceptor maintenance and function (29). To study the possible involvement of lebercilin in the transport of these proteins, we immunohistologically examined the most abundant phototransduction proteins in retinas from $L c a 5^{g t / g t}$ and $L c a 5^{+/+}$mice. We observed mislocalization of cone and rod opsins to the ISs and ONL (Figure 5A). In response to light, both arrestin and transducin partially mislocalized (Figure 5B) without affecting the localization of IFT proteins (Figure 5C), functional structural components, such as Rp1 or gamma-tubulin (Supplemental Figure 5B), or ciliary disease proteins, such as Nphp4 or Rpgrip1 (Supplemental Figure 5C). The loss of transport of opsins, as well as the reduced translocation of arrestin upon light stimulation, point to a role of lebercilin as a molecular scaffold, connecting the IFT core machinery to proteins involved in selecting and recruiting cargo. These data show that inactivation of Lca5 leads to a dose-dependent loss of ciliary IFT function in photoreceptors.

\section{Discussion}

LCA is a genetically very heterogeneous disorder, similar to most inherited retinopathies. Nevertheless, it is clearly distinguished by its congenital onset and devastatingly rapid progression. Despite this rapid progression resulting in absence of measurable ERG signals before the age of 1 year, different studies of affected human retinas by optical coherence tomography have suggested that even up to early adulthood, some ONL is retained and could be detected (30). Although an integral comparison by optical coherence tomography of all LCA subtypes is not yet available, studies have shown that at an early age, patients with mutations in genes encoding proteins present at the photoreceptor CC - such as RPGRIP1, CEP290, and lebercilin - show some preservation of the retinal microanatomic structures required for vision: photoreceptor cells, inner retina, and RPE (31-33). For the nondividing retinal cells, this is a prerequisite for development of therapeutic approaches aimed at restoration of retinal function, such as gene replacement therapy (1).

Although it was tempting to speculate that disruption of the LCA-associated proteins at the CC abrogates the bidirectional transport across the ciliary axoneme, the presence of more than 1,000 different protein species associated with this structure (34) allows many options for mechanisms that could be affected and ultimately lead to faulty ciliary transport. Our previous analysis of the lebercilin interactome by SF-TAP identified 24 interacting proteins, many of them known to be associated with microtubules and/or to have important centrosomal or ciliary functions (4). However, even combined with recent yeast-2-hybrid and coimmunoprecipitation data demonstrating the interaction of lebercilin with ciliopathy proteins such as OFD1 (35), these data did not explain the retinal disease phenotype. As we showed here, it required the increased sensitivity of the quantitative SILAC procedure in combination with efficient AP and the sensitive LTQ Orbitrap mass spectrometer to achieve detailed insight into the lebercilin interactome, which we found to contain connections to IFT complex A and B particles. Fully in line with the disrupted transport hypothesis, loss of these connections was caused by LCA5-associated mutations as a consequence of carboxyterminal truncation. Thus, analyzing the effect of mutation on a protein network level offers great prospects for functional assessment of genetic variants of unknown pathogenicity that are currently identified in high numbers with next-generation sequencing efforts of various genetic traits (36).

As a result of their inability to connect to their cognate partners on the interactome level, lebercilin mutants failed to support ciliary transport of proteins necessary for OS formation and function. In mice and humans, this results in an LCA-type early-onset blinding phenotype caused by incomplete OS development followed by photoreceptor loss. Rapid photoreceptor degeneration, accompanied by OS morphogenesis defects and opsin mislocalization in IS, as observed in retina of $L c a 5^{g t / g t}$ mice, overlaps with retinal data from Rpgrip1 mutant mice (Rpgrip ${ }^{\text {nmf247 }}$; ref. 12), Сep290 mutant mice (rd16; ref. 13), and mutant mouse models displaying defective ciliary transport (Kif3 $\mathrm{a}^{-/-}$and $\mathrm{Tg} 737^{\text {orpk }}$; refs. 37, 38). These latter data, in combination with our observed loss of all IFT and IFTassociated proteins from the lebercilin interactome when LCAcausative mutations in LCA5 were introduced, strongly support the concept that loss of the physical association of LCA-associated proteins with IFT modules is a major cause of the development of LCA. Based on the present results, we hypothesize lebercilin acts as an integral part of vesicular transport through the cilium of retinal photoreceptors. As a consequence of perturbed molecular interconnectivity between lebercilin and the IFT machinery, ciliary trafficking of lebercilin and its associated proteins via directed protein transport between ISs and OSs is likely to be affected, resulting in dysfunctional photoreceptors. Inactivation of $L c a 5$ in mice supports this scenario and underscores a role of lebercilin in ciliary transport in vivo.

Studies in vertebrates using knockout, hypomorphic mutants or knockdown of IFT proteins have shown that the proper expression of all IFT proteins appears necessary for cilium assembly and/or maintenance. However, the functional effects on this organelle differ. Whereas ift 88 mutant zebrafish fail to form OSs, ift57 mutant zebrafish form short OSs, with reduced amounts of opsin (39). Both Lca5 inactivation in vivo and siRNA-mediated knockdown of LCA5 in vitro revealed that lebercilin did not affect cilium assembly or ciliary localization of IFT88, which indicates that lebercilin is not a structural component of IFT particles. This is fully in line with the current knowledge that mutations in lebercilin do not lead to systemic ciliopathy phenotypes, but to rapid and restricted degeneration of the photoreceptors only. Specific functional constraints, dose dependency, and mechanisms of compensation may restrict LCA caused by LCA5 mutations as a ciliary disease to a single organ, whereas defects in other nodes of the ciliary network may cause a less-restricted form of ciliopathy. 
A

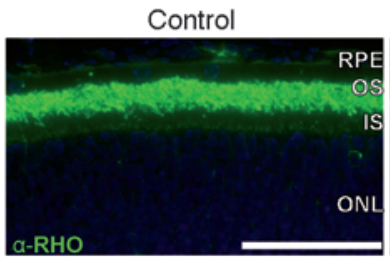

Lca5 $5^{\text {gtgt }}$
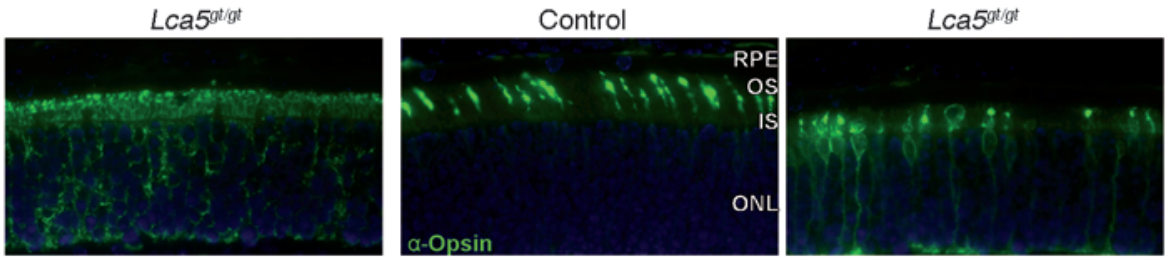

B

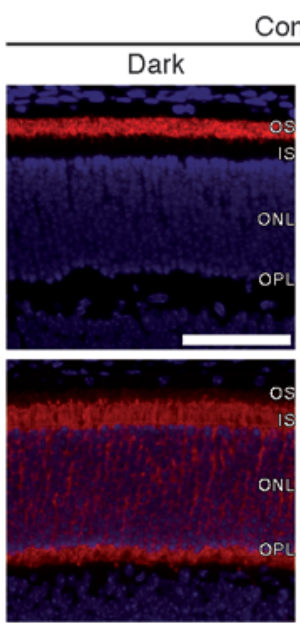

Control

Light
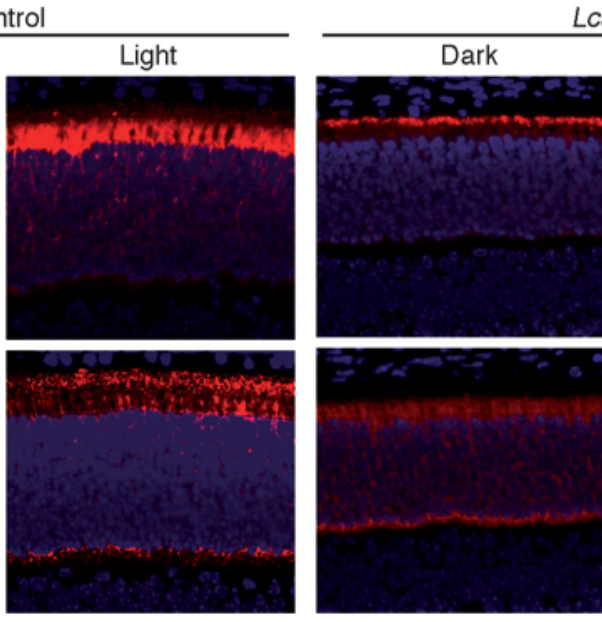

Lca5 $^{\text {thgt }}$

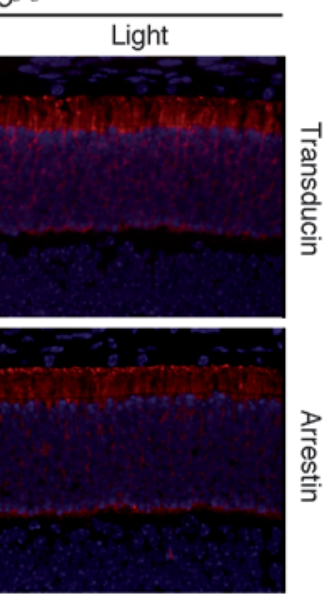

C

Control
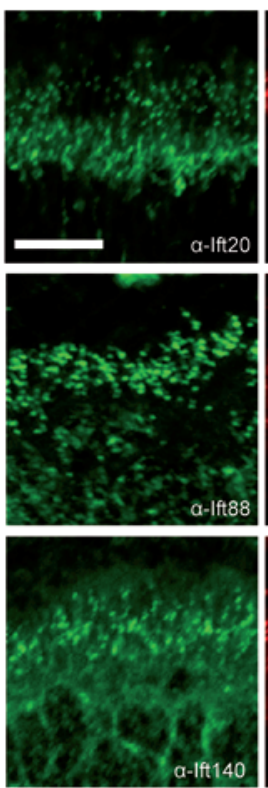
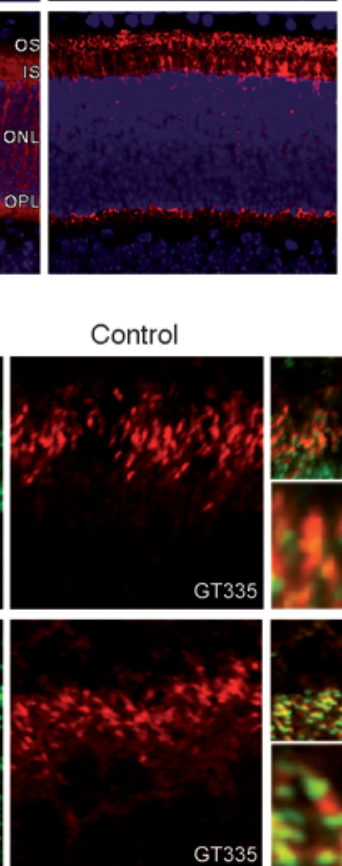

GT335

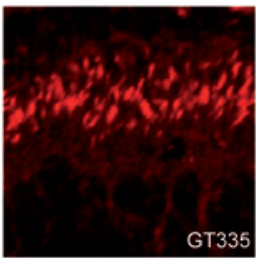

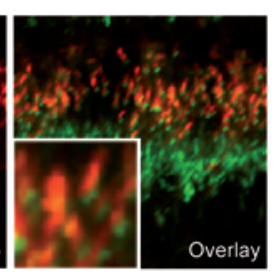
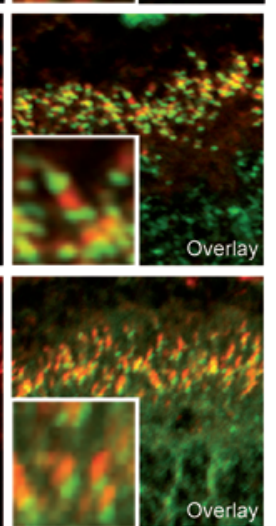
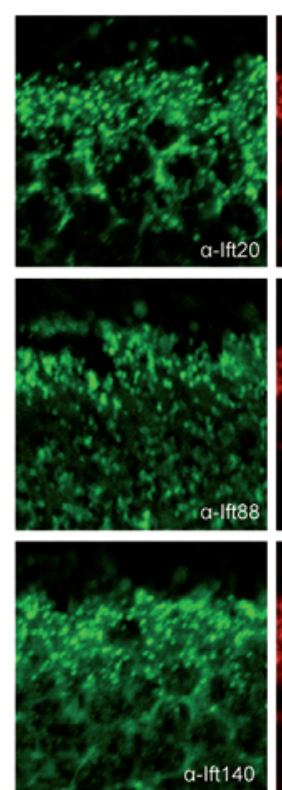

Lca $5^{\text {th } g t}$
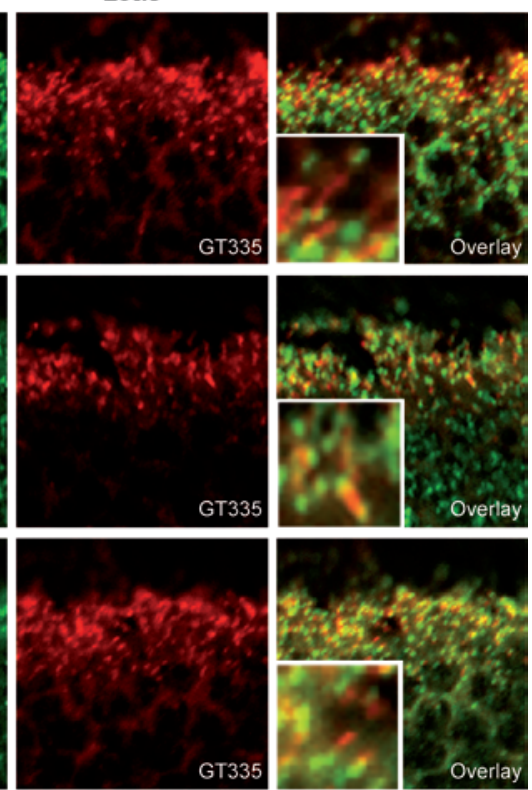

\section{Figure 5}

Localization study of phototransduction and IFT proteins in Lca5gt/gt retinas. (A) OS proteins rhodopsin (RHO) and cone opsin were mislocalized to ISs and ONL in Lca5gt/gt animals, while normally restricted to OSs in controls. All sections were obtained at P14. (B) Localization of transducin and arrestin in dark-adapted and light-stimulated animals (P14). In dark-adapted wild-type animals, transducin localized to OSs and arrestin to ISs, ONL, and outer plexiform layer (OPL). After light stimulation, transducin was mainly found in the ISs, with weaker staining in the ONL and outer plexiform layer, whereas arrestin translocated to the OSs. In Lca5 ${ }^{\text {gt/gt }}$ dark-adapted animals, the majority of transducin was found in the OSs, with weaker staining in other layers. Arrestin localization in Lca5t/gt dark-adapted animals was similar to that in dark-adapted wild-type mice. In light-stimulated Lca59t/gt mice, transducin not only was found in the ISs, but also showed diffuse staining in the entire ONL. Arrestin staining appeared diffusely throughout the photoreceptor layer, with less pronounced OS staining than in wild-type mice. (C) Localization of Ift20, Ift88, or Ift140 in connecting cilia of photoreceptors was not affected by inactivation of Lca5 compared with wild-type at P9. GT335 specifically stained connecting cilia. Scale bars: $50 \mu \mathrm{m}(\mathbf{A}$ and $\mathbf{B}) ; 10 \mu \mathrm{m}(\mathbf{C})$. Enlarged views are shown in the insets $(\mathbf{C}, \times 3)$. 
Our findings, combined with the fact that proteins mutated in Bardet-Biedl syndrome (40), Jeune syndrome (41), retinitis pigmentosa (42), or Sensenbrenner syndrome $(43,44)$ localize to or constitute IFT particles, strongly suggest that loss of IFT causes photoreceptor degeneration in these diseases as well. On a systemic level, these diseases are connected through a joint network of protein interactions perturbed as a consequence of mutation. Members of this interactome are likely candidate genes for other ciliopathies and transport disorders yet to be discovered.

\section{Methods}

Generation of gene trap animals and husbandry. ES cells containing a Lca5 gene trap $\left(L c a 5^{t t}\right)$ were obtained from Sanger Institute Gene Trap Resources (SIGTR; ES cell line AG0283) through Mutant Mouse Regional Research Centers (MMRRC) and injected into C57BL/6J (B6) blastocysts. Detailed methods for microinjection and mutant selection were described previously (45). The injected cells were transferred to pseudopregnant female B6 mice to obtain chimeras. The chimeric males (F1) genotyped for the Lca5 gene trap allele $\left(\mathrm{Lca5}^{+/ g t}\right)$ were crossed to B6 females, and the subsequent gene trap-positive progeny were intercrossed. All results in this study were obtained from F2 animals. Mice were maintained in a vivariam with a 12-hour light/12-hour dark cycle and fed a $4 \%$ NIH diet and acidified water. For the arrestin/transducin translocation studies, mice were dark-adapted for 16 hours, and the eyes were harvested under red light. For light stimulation, mice were transferred to a box lined with mirrors and exposed to approximately 1,500 lux of light after their pupils were dilated with atropine. Whole eyes were collected 30 minutes after light exposure. Animals were genotyped using I3F5, I3R1, and En2.6R primers. Primer sequences are shown in Supplemental Table 5. Genomic DNA was isolated from tail tip, and PCR amplicons were obtained and visualized as previously described (12). Procedures using mice were approved by The Jackson Laboratory Institutional Animal Care and Use Committee.

Ophthalmic examination and histology. The methods for fundus photography and ERG have been described previously (12). For histological examination by light microscopy, whole eyes were collected and fixed overnight in methanol and acetic acid $(3: 1, v / v)$, washed, and embedded in paraffin. Tissue samples were cut in 4 - to $6-\mu \mathrm{m}$ sections and stained with hematoxylin and eosin. For transmission EM, the cornea and lens were removed, and the posterior retina was fixed overnight in $2.5 \%$ glutaraldehyde and $2 \%$ PFA. For scanning EM, retinas were dissected and fixed overnight in $3 \%$ glutaraldehyde and 1\% PFA. Both transmission EM and scanning EM samples were postfixed with osmium tetroxide and stained with uranyl acetate as previously described (12).

Cell culture. Human B lymphocytes from a LCA patient (family 27240; ref. 4) and an unaffected control were immortalized by transformation with EBV according to established procedures (46) and grown as described previously (35). This study was approved by the Research Ethics Board of McGill University. We obtained blood samples after receiving informed consent from all participating individuals. hTERT-RPE1 cells and HEK293T cells were cultured as described previously (47). For SILAC experiments, HEK293T cells were grown in SILAC DMEM (PAA) supplemented with $3 \mathrm{mM}$ L-glutamine (PAA), 10\% dialyzed fetal bovine serum (PAA), $0.55 \mathrm{mM}$ lysine, and $0.4 \mathrm{mM}$ arginine. Light SILAC medium was supplemented with ${ }^{12} \mathrm{C}_{6},{ }^{14} \mathrm{~N}_{2}$ lysine and ${ }^{12} \mathrm{C}_{6},{ }^{14} \mathrm{~N}_{4}$ arginine. Heavy SILAC medium was supplemented with either ${ }^{13} \mathrm{C}_{6}$ lysine and ${ }^{13} \mathrm{C}_{6},{ }^{15} \mathrm{~N}_{4}$ arginine or ${ }^{13} \mathrm{C}_{6},{ }^{15} \mathrm{~N}_{2}$ lysine and ${ }^{13} \mathrm{C}_{6},{ }^{15} \mathrm{~N}_{4}$ arginine. $0.5 \mathrm{mM}$ proline was added to all SILAC media to prevent arginine-to-proline conversion (48). All aa were purchased from Silantes. Primary cilium formation in hTERT-RPE1 cells was induced by serum starvation $(0.2 \%$ serum) for 48 hours. For siRNA experiments, hTERT-RPE1 cells were seeded on glass slides, grown over- night, and then transfected with 3 pooled Silencer Select siRNAs (15 nM final siRNA concentration; Ambion) using Hiperfect (Qiagen) according to the manufacturer's instructions. Nontargeting siRNA control no. 1 (15 nM final siRNA concentration; Ambion) was included as reference. The targeting sequence for each siRNA is included in Supplemental Table 4. After 72 hours of siRNA transfection, cells were harvested for either knockdown analysis or immunofluorescence. For DNA transfections, HEK293T or hTERT-RPE1 cells were seeded, grown overnight, and then transfected using Effectene (Qiagen) according to the manufacturer's instructions.

DNA constructs. We generated IFT cDNA constructs by PCR using IMAGE clones of IFT20 (clone 3609552), IFT27 (clone 3163341), IFT57 (clone 4053749), and IFT88 (clone 4824965; all from Geneservice) cDNA as template. IFT52 was generated via PCR from human marathon-ready testis cDNA (Clontech). Gateway-adapted constructs were created using the Gateway cloning system (Invitrogen) according to the manufacturer's procedures and were sequence verified. Gateway-adapted full-length human lebercilin, lebercilin-p.P493TfsX1, and lebercilin-p.Q279X have been described previously $(4,49)$.

AP of protein complexes. For 1-step Strep purifications, SF-TAP-tagged proteins and associated protein complexes were purified essentially as described previously $(4,14)$. HEK293T cells transiently expressing the SF-TAP tagged constructs were lysed in lysis buffer containing $0.5 \%$ Nonidet-P40, protease inhibitor cocktail (Roche), and phosphatase inhibitor cocktails I and II (Sigma-Aldrich) in TBS (30 mM Tris- $\mathrm{HCl}, \mathrm{pH}$ 7.4 , and $150 \mathrm{mM} \mathrm{NaCl}$ ) for 20 minutes at $4^{\circ} \mathrm{C}$. After sedimentation of nuclei at $10,000 \mathrm{~g}$ for 10 minutes, the cleared lysates were transferred to Strep-Tactin-Superflow beads (IBA) and incubated for 1 hour before the resin was washed 3 times with wash buffer (TBS containing 0.1\% NP40 and phosphatase inhibitor cocktails I and II). The protein complexes were eluted by incubation for 10 minutes in Strep-elution buffer (IBA). For quantitative protein complex comparison or detection of specific complex components, protein concentration was determined using a standard Bradford assay. Equal protein amounts were used as input for the experiments to be compared. The protein complex purification was done as described above with 1 modification: the samples were combined before the resin was washed and the protein complexes were eluted. The eluted samples were concentrated using 10-kDa cutoff VivaSpin 500 centrifugal devices (Sartorius Stedim Biotech) and prefractionated using SDS-PAGE and in-gel tryptic cleavage as described elsewhere (47). For SF-TAP analysis, the constructs were expressed and cells were harvested as described above. The cleared supernatant was incubated for 1 hour at $4^{\circ} \mathrm{C}$ with Strep-Tactin superflow (IBA). Subsequently, the resin was washed 3 times in wash buffer. Protein baits were eluted with Strep-elution buffer. For the second purification step, the eluates were transferred to anti-FlagM2 agarose (Sigma-Aldrich) and incubated for 1 hour at $4^{\circ} \mathrm{C}$. The beads were washed 3 times with wash buffer, and proteins were eluted with Flag peptide ( $200 \mu \mathrm{g} / \mathrm{ml}$; Sigma-Aldrich) in TBS. After purification, the samples were precipitated with chloroform and methanol and subjected to in-solution tryptic cleavage as described previously (47).

Mass spectrometry and data analysis. LC-MS/MS analysis was performed on an Ultimate3000 nano HPLC system (Dionex) coupled to a LTQ OrbitrapXL mass spectrometer (Thermo Fisher Scientific) by a nano spray ion source. Tryptic peptide mixtures were automatically injected and loaded at a flow rate of $30 \mu \mathrm{l} / \mathrm{min}$ in $95 \%$ buffer $\mathrm{C}(0.5 \%$ trifluoroacetic acid in HPLC-grade water) and 5\% buffer B (98\% actetonitrile and $0.1 \%$ formic acid in HPLC-grade water) onto a nano trap column $(100 \mu \mathrm{m}$ i.d. $\times 2 \mathrm{~cm}$, packed with Acclaim PepMap100 C18, 5 um, 100 Å; Dionex). After 5 minutes, peptides were eluted and separated on the analytical column $(75 \mu \mathrm{m}$ i.d. $\times 15$ cm, Acclaim PepMap100 C18, $3 \mu \mathrm{m}, 100 \AA \AA$; Dionex) by a linear gradient from $5 \%$ to $40 \%$ of buffer B in buffer $\mathrm{A}$ ( $2 \%$ acetonitrile and $0.1 \%$ 
formic acid in HPLC-grade water) at a flow rate of $300 \mathrm{nl} /$ min over 90 minutes. Remaining peptides were eluted by a short gradient from $40 \%$ to $100 \%$ buffer B in 5 minutes. The eluted peptides were analyzed by the LTQ OrbitrapXL mass spectrometer. From the high-resolution mass spectrometry prescan with a mass range of $300-1,500$, the 10 most intense peptide ions were selected for fragment analysis in the linear ion trap if they exceeded an intensity of at least 200 counts and if they were at least doubly charged. The normalized collision energy for collision-induced dissociation was set to a value of 35 , and the resulting fragments were detected with normal resolution in the linear ion trap. The lock mass option was activated, the background signal with a mass of 445.12002 was used as lock mass (50). Every ion selected for fragmentation was excluded for 30 seconds by dynamic exclusion. For SILAC experiments, all acquired spectra were processed and analyzed using MaxQuant software (version 1.0.13.13; ref. 16) and the human-specific IPI database version 3.52 (http://www.maxquant.org/) in combination with Mascot (version 2.2; Matrix Science). Cysteine carbamidomethylation was selected as fixed modification; methionine oxidation and protein acetylation were allowed as variable modifications. The peptide and protein false discovery rates were set to $1 \%$. Contaminants like keratins were removed. Proteins identified and quantified by at least 2 peptides per experiment in at least 2 of 3 independent experiments were considered for further analysis. Each experiment consisted of a forward and a reverse labeling approach to exclude label-specific effects. A $P$ value of 0.001 was selected as threshold for significant enrichment or alteration. Only proteins identified as specific lebercilin complex components by significant enrichment in the comparison of wild-type lebercilin with SF-TAP control were considered for the quantitative protein complex comparisons. As there is little relevant information present in public databases, including STRING, MINT, and IntAct, we annotated and grouped protein interactions manually based on publicly available data and constructed a scale-free protein interaction map representing proteins as nodes and interactions as edges.

For nonquantitative experiments, the raw data were analyzed using Sequest (Thermo Fisher Scientific) or Mascot and Scaffold (Proteome Software) as described previously (47). Proteins were considered to be specific protein complex components if they were not detected in the control and were detected at least twice with 2 or more peptides (peptide probability $>80 \%$ ) in 3 experiments. The protein probability threshold was set to $99 \%$.

Northern blot analysis. Total retinal RNA was isolated from wildtype, heterozygous, and homozygous Lca $5^{g t}$ mice at P17 using TRIzOL (Invitrogen) reagents, separated in a $1 \%$ formamide gel by electrophoresis, and transferred onto a nitrocellulose membrane. The $\mathrm{N}$-terminal probe for Lca5 (860 bp) was generated by RT-PCR amplification using primers E2F1 and E4R1 (see Supplemental Table 5). 18S control probe (Ambion) and $L c a 5$ probe were labeled with ${ }^{35} \mathrm{P}$ using the Redi-prime II DNA labeling kit (Amersham) and incubated with the membrane overnight at $42^{\circ} \mathrm{C}$. The resulting images were obtained by autoradiography.

$\beta$-Galactosidase assay. Dissected retina, brain, and testis were incubated in PBS containing $30 \mathrm{mM} \mathrm{K}_{4} \mathrm{Fe}(\mathrm{CN})_{6}, 30 \mathrm{mM} \mathrm{K}_{3} \mathrm{Fe}(\mathrm{CN})_{6}, 2 \mathrm{mM} \mathrm{MgCl}_{2}, 0.01 \%$ sodium desoxycholate, and $0.02 \% \mathrm{NP}-40$ after 3 hours of fixation with $4 \%$ PFA. Additional fixation with fresh fixative overnight was carried out after the blue color developed in the tissue expressing $L c a 5^{g t}$. The stained tissues were photographed and embedded in paraffin. $6-\mu \mathrm{m}$ sections were stained with Nuclear Fast Red for nuclear counterstaining.

$R T-P C R$. The gene trap insertion site was identified by direct sequencing of the RT-PCR amplicon with primers E3F1 and en2R1 from ES cell cDNA. Retinal cDNA from $L c a 5^{+/+}$and $L c a 5^{g t / g t}$ animals was generated using the Retroscript kit (Ambion). Primers used for Lca 5 cDNA analysis (E2F1, E4R1, E3F2, and LacZ6R) and input cDNA control (rig/S15; Ambion) are included in Supplemental Table 5.
Quantitative PCR. Total RNA from ciliated hTERT-RPE1 cells and EBVtransformed cell lines was isolated using TRIzOL (Invitrogen) according to the manufacturer's instructions. Residual traces of genomic DNA were removed via DNase I (Invitrogen) treatment according to the manufacturer's instructions. $1 \mu \mathrm{g}$ total RNA was transcribed into cDNA using the iScript cDNA synthesis kit (BioRad Laboratories) according to the manufacturer's protocol. SYBR Green-based qPCR analysis was performed on a 7500 Fast Real-Time PCR System (Applied Biosystems) with Power SYBR Green PCR Master Mix (Applied Biosystems) according to the manufacturer's instructions. Primers were developed by the Primer3 program (Supplemental Table 5 and ref. 51). PCR products encompassed at least 1 exon-exon junction. GUSB was used as reference gene. Further analysis was performed as described previously (35).

GST pulldown. In order to produce GST fusion proteins, BL21-DE3 cells were transformed with either pDEST15-lebercilin or pDEST15 alone. Cells were induced at $30^{\circ} \mathrm{C}$ for 3 hours with $0.5 \mathrm{mM}$ IPTG (Sigma-Aldrich) and subsequently lysed with STE buffer (10 mM Tris-HCl, pH 8.0; 1 mM EDTA; and $150 \mathrm{mM} \mathrm{NaCl}$ ) supplemented with $10 \mathrm{mg} / \mathrm{ml}$ lysozyme, $0.5 \%$ Sarkosyl, $1 \%$ Triton X-100, and complete protease inhibitor cocktail (Roche). Lysates were incubated for 2 hours at $4^{\circ} \mathrm{C}$ with glutathione-sepharose $4 \mathrm{~B}$ beads (Amersham Biosciences). After incubation, beads were washed with STE and TBSTD (TBS with $1 \%$ Triton X-100 and $2 \mathrm{mM} \mathrm{DTT)}$ ). The amount of GST fusion proteins bound to the beads was verified on a NuPAGE Novex 4\%-12\% Bis-Tris SDS-PAGE gel by staining with SimplyBlue SafeStain (Invitrogen). Beads with bound GST fusion proteins were incubated for 2.5 hours at $4{ }^{\circ} \mathrm{C}$ with bovine retinal lysates, made by incubating retina in $50 \mathrm{mM}$ Tris- $\mathrm{HCl}$ (pH 7.4), $150 \mathrm{mM} \mathrm{NaCl}, 10 \%$ glycerol, and 1\% Triton-X100 supplemented with complete protease inhibitors (Roche). Beads with bound protein complexes were washed in lysis buffer, then taken up in $1 \times$ NuPage Sample Buffer, heated for 10 minutes at $70^{\circ} \mathrm{C}$, precipitated by centrifugation, and subjected to immunoblot analysis.

Immunochemistry. Mouse retinal sections were obtained either from paraffin-embedded eyes or from fresh-frozen sections, washed in $0.01 \%$ Tween-20/PBS for 20 minutes, blocked overnight at $4{ }^{\circ} \mathrm{C}$ with blocking solution $(0.1 \%$ ovalbumin and $0.5 \%$ fish gelatin in PBS), and incubated for 1 hour with antibodies in the presence of blocking solution. Ciliated hTERT-RPE1 cells were washed briefly in PBS, fixed in 2\% PFA/PBS for 10 minutes, treated with $1 \%$ Triton-X100/PBS for 5 minutes, and blocked with $2 \% \mathrm{BSA} / \mathrm{PBS}$ for 30 minutes. Mouse retinal sections and fixed hTERT-RPE1 cells were stained using anti-rhodopsin (1:500, Leico Technologies), anti-red/green cone opsin (1:250, Chemicon), anti-transducin (1:200, Santa Cruz Biotechnology Inc.), anti-arrestin (1:1,000, ABR), anti-NPHP4 (1:200, raised against $\mathrm{N}$-terminal recombinant protein), antiRPGRIP1 (1:1,000, provided by T. Li, National Eye Institute, Bethesda, Maryland, USA), anti-RP1 (1:2,000, gift of E. Pierce, Scheie Eye Institute, Philadelphia, Pennsylvania, USA), anti- $\gamma$-tubulin (1:500, Sigma-Aldrich), GT335 (1:1,000, detecting polyglutamylated tubulin, mouse monoclonal; provided by C. Janke, Institut Curie, Orsay, France), anti-acetylated tubulin (1:1,000, Sigma-Aldrich), anti-IFT88 (1:300, Abnova; 1:300, provided by G. Pazour, University of Massachusetts Medical School, Worcester, Massachusetts, USA), anti-TRAF3IP1 (1:300, Abnova), anti-WDR19 (1:300, Abnova), anti-IFT20 and anti-IFT140 (1:300, provided by G. Pazour), anti-RPGRIP1L (1:1,000, SNC040; ref. 52) or anti-lebercilin (1:300, ref. 4; 1:500, SN2134). Rabbit polyclonal antisera to lebercilin (SN2134 and SN2135) were raised against purified C-terminal human lebercilin (aa residues 491-697) by Eurogentec. Following primary antibody incubation, cells and sections were subsequently washed with PBS and incubated for 45 minutes with either Cy3-conjugated (1:250, Jackson Immunoresearch Laboratories) or Alexa Fluor 405, 488, or 568 (1:500, Invitrogen) secondary antibodies and embedded in either Prolong Gold Anti-fade 
(Invitrogen) for retinal sections or Vectashield with DAPI (Vector Laboratories) for hTERT-RPE1 cells. Cells and retinal sections were analyzed with a Zeiss Axio Imager Z1 fluorescence microscope (Carl Zeiss). Optical sections were generated through structured illumination by inserting an ApoTome slider into the illumination path, followed by processing with AxioVision (Carl Zeiss) and Photoshop CS4 software (Adobe Systems).

Immunoblot analysis. Dissected retinas were lysed in NP-40 lysis buffer with sodium deoxycholate and EDTA, then supplemented with complete protease inhibitors (Roche). For protein analysis of TAP eluates, cells were lysed in lysis buffer containing $0.5 \%$ Nonidet-P40, protease inhibitor cocktail (Roche), and phosphatase inhibitor cocktails I and II (Sigma-Aldrich) in TBS ( $30 \mathrm{mM}$ Tris- $\mathrm{HCl}, \mathrm{pH} 7.4$, and $150 \mathrm{mM} \mathrm{NaCl}$ ) for 20 minutes at $4{ }^{\circ} \mathrm{C}$, and nuclei and cell debris were sedimented by centrifugation at $10,000 \mathrm{~g}$ for 10 minutes at $4{ }^{\circ} \mathrm{C}$. Equal protein amounts of lysates or eluates were separated by SDS-PAGE and electrophoretically transferred to nitrocellulose membranes. Membranes were blocked in 5\% nonfat milk in TBS containing $0.1 \%$ Tween 20 (Sigma-Aldrich) and incubated with anti-lebercilin (1:500, SN2135 and 1702\#2, rabbit polyclonal antibody mix raised using multiple synthetic peptides covering aa 1-20, 493-512, and 667-686 of mouse lebercilin; Peptide Specialty Laboratories GmbH), anti- $\gamma$-tubulin (1:2,000, Sigma-Aldrich), anti-IFT74 (1:300, Everest Biotech), anti-IFT88 and anti-IFT57 (1:500, gifts of G. Pazour), anti-TRAF3IP1 (1:500, Abnova), anti-14-3-3 $\varepsilon$ (1:1,000, Santa Cruz Biotechnology Inc.), anti-GST (1:1,000, Sigma-Aldrich), and anti-FlagM2 (1:1,000, Sigma-Aldrich). Secondary antibodies from Jackson Immunoresearch were used $(1: 15,000)$. Protein bands were visualized by ECL plus (GE Healthcare).

Statistics. Quantitative mass spectrometric data were automatically analyzed by MaxQuant software. The outlier (2-tailed) significance score for $\log$ protein ratios (significance $\mathrm{A}$ ) and $P$ values for detection of a significant outlier from the main distribution were automatically calculated by MaxQuant. Details for MaxQuant-based statistical analyses have been described previously (16). For protein complex detection of wild-type lebercilin, only enriched proteins (enrichment ratio $>1$ versus SF-TAP control) were considered. For the protein complex comparisons of both LCA-associated lebercilin mutants, both stronger and weaker binding interactors compared with wild-type lebercilin were considered. MaxQuant-based significance A values with $P<0.001$ were considered significant for all experiments.

\section{Acknowledgments}

This work was supported by the National Genome Research Framework program NGFN-Plus; the Helmholtz Alliance for Mental Health in an Aging Society (HelMA; to M. Ueffing); the European Community's Seventh Framework Programme FP7/2009 under grant agreement no. 241955; SYSCILIA (to R. Roepman and M. Ueffing); the German Federal Ministry of Education and Research (BMBF, DYNAMO; FKZ, 0315513A; to M. Ueffing); the Kerstan Foundation (to M. Ueffing); grants from the Netherlands Organization for Scientific Research (NWO Vidi-91786396; to R. Roepman); the Foundation for Retinal Research (to A.I. den Hollander, R.K. Koenekoop, J. Bennett, F.P.M. Cremers, P.M. Nishina, and R. Roepman); the Foundation Fighting Blindness (to P.M. Nishina, R.K. Koenekoop, and R. Roepman); and institutional grant CA34196 to The Jackson Laboratory. We thank Chantal Longo-Guess and Kenneth Johnson for ABR tests of hearing function, Moniek Riemersma for help in cloning IFT constructs, Emine Bolat for qPCR assistance, Heleen Arts for help in lebercilin antibody production, and Theo Peters for retinal sectioning. We thank Bernd Wissinger and Nicholas Katsanis for critical reading of the manuscript. We thank Sandra Helm, Silke Becker, and Hakan Sarioglu from the Core Facility Proteomics at Helmholtz-Zentrum München for their excellent technical support.

Received for publication November 1, 2010, and accepted in revised form April 12, 2011.

Address correspondence to: Marius Ueffing, Division of Experimental Ophthalmology, Centre for Ophthalmology, Institute for Ophthalmic Research, University of Tübingen, Röntgenweg 11, 72076 Tübingen, Germany. Phone: 49.7071.29.84021; Fax: 49.7071.29.4560; E-mail: marius.ueffing@uni-tuebingen.de. Or to: Ronald Roepman, Department of Human Genetics (855), Radboud University Nijmegen Medical Centre, P.O. Box 9101, 6500 HB Nijmegen, Netherlands. Phone: 31.24.3610487; Fax: 31.24.3668752; E-mail: r.roepman@antrg.umcn.nl.
1. den Hollander AI, Black A, Bennett J, Cremers FP. Lighting a candle in the dark: advances in genetics and gene therapy of recessive retinal dystrophies. J Clin Invest. 2010;120(9):3042-3053.

2. Estrada-Cuzcano A, et al. IQCB1 mutations in patients with Leber congenital amaurosis. Invest Ophthalmol Vis Sci. 2011;52(2):834-839.

3. den Hollander AI, Roepman R, Koenekoop RK, Cremers FP. Leber congenital amaurosis: genes, proteins and disease mechanisms. Prog Retin Eye Res. 2008;27(4):391-419.

4. den Hollander AI, et al. Mutations in LCA5, encoding the ciliary protein lebercilin, cause Leber congenital amaurosis. Nat Genet. 2007;39(7):889-895.

5. Blacque OE, Cevik S, Kaplan OI. Intraflagellar transport: from molecular characterisation to mechanism. Front Biosci. 2008;13:2633-2652.

6. Qin H, Diener DR, Geimer S, Cole DG, Rosenbaum JL. Intraflagellar transport (IFT) cargo: IFT transports flagellar precursors to the tip and turnover products to the cell body. J Cell Biol. 2004; 164(2):255-266.

7. Rosenbaum JL, Witman GB. Intraflagellar transport. Nat Rev Mol Cell Biol. 2002;3(11):813-825.

8. Scholey JM. Intraflagellar transport. Annu Rev Cell Dev Biol. 2003;19:423-443.

9. Piperno G, Siuda E, Henderson S, Segil M, Vaananen $\mathrm{H}$, Sassaroli M. Distinct mutants of retrograde intraflagellar transport (IFT) share similar morphological and molecular defects. J Cell Biol. 1998;143(6):1591-1601.

10. Piperno G, Mead K. Transport of a novel complex in the cytoplasmic matrix of Chlamydomonas flagella. Proc Natl Acad Sci U S A. 1997;94(9):4457-4462.

11. Young RW. The renewal of photoreceptor cell outer segments. J Cell Biol. 1967;33(1):61-72.

12. Won J, et al. RPGRIP1 is essential for normal rod photoreceptor outer segment elaboration and morphogenesis. Hum Mol Genet. 2009;18(22):4329-4339.

13. Chang B, et al. In-frame deletion in a novel centrosomal/ciliary protein CEP290/NPHP6 perturbs its interaction with RPGR and results in early-onset retinal degeneration in the $\mathrm{rd} 16$ mouse. Hum Mol Genet. 2006;15(11):1847-1857.

14. Gloeckner CJ, Boldt K, Schumacher A, Roepman $\mathrm{R}$, Ueffing $M$. A novel tandem affinity purification strategy for the efficient isolation and characterisation of native protein complexes. Proteomics. 2007;7(23):4228-4234.

15. Ong SE, et al. Stable isotope labeling by amino acids in cell culture, SILAC, as a simple and accurate approach to expression proteomics. Mol Cell Proteomics. 2002;1(5):376-386

16. Cox J, Mann M. MaxQuant enables high peptide identification rates, individualized p.p.b.-range mass accuracies and proteome-wide protein quantification. Nat Biotechnol. 2008;26(12):1367-1372.

17. Cole DG, Diener DR, Himelblau AL, Beech PL, Fus- ter JC, Rosenbaum JL. Chlamydomonas kinesin-IIdependent intraflagellar transport (IFT): IFT particles contain proteins required for ciliary assembly in Caenorhabditis elegans sensory neurons. J Cell Biol. 1998;141(4):993-1008.

18. Adhiambo C, Blisnick T, Toutirais G, Delannoy E, Bastin P. A novel function for the atypical small $G$ protein Rab-like 5 in the assembly of the trypanosome flagellum. J Cell Sci. 2009;122(pt 6):834-841.

19. Blacque OE, et al. Functional genomics of the cilium, a sensory organelle. Curr Biol. 2005;15(10):935-941.

20. Li C, et al. An essential role for DYF-11/MIP-T3 in assembling functional intraflagellar transport complexes. PLoS Genet. 2008;4(3):e1000044.

21. Ou G, Blacque OE, Snow JJ, Leroux MR, Scholey JM. Functional coordination of intraflagellar transport motors. Nature. 2005;436(7050):583-587.

22. Schafer JC, Winkelbauer ME, Williams CL, Haycraft CJ, Desmond RA, Yoder BK. IFTA-2 is a conserved cilia protein involved in pathways regulating longevity and dauer formation in Caenorhabditis elegans. J Cell Sci. 2006;119(pt 19):4088-4100.

23. Murayama T, Toh Y, Ohshima Y, Koga M. The dyf3 gene encodes a novel protein required for sensory cilium formation in Caenorhabditis elegans. $J \mathrm{Mol}$ Biol. 2005;346(3):677-687.

24. Omori Y, et al. Elipsa is an early determinant of ciliogenesis that links the IFT particle to membrane-associated small GTPase Rab8. Nat Cell Biol. 
2008;10(4):437-444

25. Wang Z, Fan ZC, Williamson SM, Qin H. Intraflagellar transport (IFT) protein IFT25 is a phosphoprotein component of IFT complex B and physically interacts with IFT27 in Chlamydomonas. PLoS One. 2009;4(5):e5384.

26. Follit JA, Xu F, Keady BT, Pazour GJ. Characterization of mouse IFT complex B. Cell Motil Cytoskeleton. 2009;66(8):457-468.

27. Behm-Ansmant I, Izaurralde E. Quality control of gene expression: a stepwise assembly pathway for the surveillance complex that triggers nonsense-mediated mRNA decay. Genes Dev. 2006;20(4):391-398.

28. Kunitomo H, Iino Y. Caenorhabditis elegans DYF-11, an orthologue of mammalian Traf3ip1/MIP-T3, is required for sensory cilia formation. Genes Cells. 2008;13(1):13-25.

29. Insinna C, Besharse JC. Intraflagellar transport and the sensory outer segment of vertebrate photoreceptors. Dev Dyn. 2008;237(8):1982-1992.

30. Pasadhika S, et al. Differential macular morphology in patients with RPE65-, CEP290-, GUCY2Dand AIPL1-related Leber congenital amaurosis. Invest Ophthalmol Vis Sci. 2010;51(5):2608-2614.

31. Cideciyan AV, et al. Centrosomal-ciliary gene CEP290/NPHP6 mutations result in blindness with unexpected sparing of photoreceptors and visual brain: implications for therapy of Leber congenital amaurosis. Hum Mutat. 2007;28(11):1074-1083.

32. Jacobson SG, et al. Leber congenital amaurosis caused by Lebercilin (LCA5) mutation: retained photoreceptors adjacent to retinal disorganization. Mol Vis. 2009;15:1098-1106.

33. Jacobson SG, et al. Leber congenital amaurosis caused by an RPGRIP1 mutation shows treatment potential. Ophthalmology. 2007;114(5):895-898.

34. Liu Q, et al. The proteome of the mouse photoreceptor sensory cilium complex. Mol Cell Proteomics. 2007;6(8):1299-1317.

35. Coene KL, et al. OFD1 is mutated in X-linked Joubert syndrome and interacts with LCA5-encoded lebercilin. Am J Hum Genet. 2009;85(4):465-481.

36. Li Y, et al. Resequencing of 200 human exomes identifies an excess of low-frequency non-synonymous coding variants. Nat Genet. 2010;42(11):969-972.

37. Pazour GJ, et al. The intraflagellar transport protein, IFT88, is essential for vertebrate photoreceptor assembly and maintenance. J Cell Biol. 2002;157(1):103-113.

38. Marszalek JR, et al. Genetic evidence for selective transport of opsin and arrestin by kinesin-II in mammalian photoreceptors. Cell. 2000;102(2):175-187.

39. Krock BL, Perkins BD. The intraflagellar transport protein IFT57 is required for cilia maintenance and regulates IFT-particle-kinesin-II dissociation in vertebrate photoreceptors. J Cell Sci. 2008;121(pt 11):1907-1915.

40. Lechtreck KF, et al. The Chlamydomonas reinhardtii BBSome is an IFT cargo required for export of specific signaling proteins from flagella. J Cell Biol. 2009;187(7):1117-1132.

41. Beales PL, et al. IFT80, which encodes a conserved intraflagellar transport protein, is mutated in Jeune asphyxiating thoracic dystrophy. Nat Genet. 2007;39(6):727-729.

42. Khanna H, et al. RPGR-ORF15, which is mutated in retinitis pigmentosa, associates with SMC1, SMC3, and microtubule transport proteins. J Biol Chem. 2005;280(39):33580-33587.

43. Gilissen C, et al. Exome sequencing identifies WDR35 variants involved in Sensenbrenner syn- drome. Am J Hum Genet. 2010;87(3):418-423.

44. Walczak-Sztulpa J, et al. Cranioectodermal Dysplasia, Sensenbrenner syndrome, is a ciliopathy caused by mutations in the IFT122 gene. Am J Hum Genet. 2010;86(6):949-956.

45. Collin GB, et al. Alms1-disrupted mice recapitulate human Alstrom syndrome. Hum Mol Genet. 2005;14(16):2323-2333

46. Wall FE, Henkel RD, Stern MP, Jenson HB, Moyer MP. An efficient method for routine Epstein-Barr virus immortalization of human B lymphocytes. In Vitro Cell Dev Biol Anim. 1995;31(2):156-159.

47. Gloeckner CJ, Boldt K, Ueffing M. Strep/FLAG tandem affinity purification (SF-TAP) to study protein interactions. Curr Protoc Protein Sci. 2009; Chapter 19:Unit19.20

48. Bendall SC, Hughes C, Stewart MH, Doble B, Bhatia M, Lajoie GA. Prevention of amino acid conversion in SILAC experiments with embryonic stem cells. Mol Cell Proteomics. 2008;7(9):1587-1597.

49. van Wijk E, et al. Usher syndrome and Leber congenital amaurosis are molecularly linked via a novel isoform of the centrosomal ninein-like protein. Hum Mol Genet. 2009;18(1):51-64.

50. Olsen JV, et al. Parts per million mass accuracy on an Orbitrap mass spectrometer via lock mass injection into a C-trap. Mol Cell Proteomics. 2005; 4(12):2010-2021.

51. Rozen S, Skaletsky H. Primer3 on the WWW for general users and for biologist programmers. Methods Mol Biol. 2000;132:365-386.

52. Arts $\mathrm{HH}$, et al. Mutations in the gene encoding the basal body protein RPGRIP1L, a nephrocystin-4 interactor, cause Joubert syndrome. Nat Genet. 2007;39(7):882-888 